\title{
TEORÍA ENFERMERA APROXIMACIÓN AL CONTEXTO ACTUAL DE ENFERMERÍA
}

Bernardo Vila Blasco*.

Concha Bilbao Guerrero**.

Antonio Porras Cabrera***.

Ana Sáez Moleón**.

Dolores Ruiz Diana**.

Daniel Lupión González**.

* Profesor Titular E. CC.de la Salud. Universidad de Málaga.

** Profesora Asociada a Tiempo Completo.

*** Profesor Asociado a Tiempo Parcial.

Departamento de Enfermería. Universidad de Málaga. Paseo de Martiricos s/n. 29009 Málaga.

\section{RESUMEN}

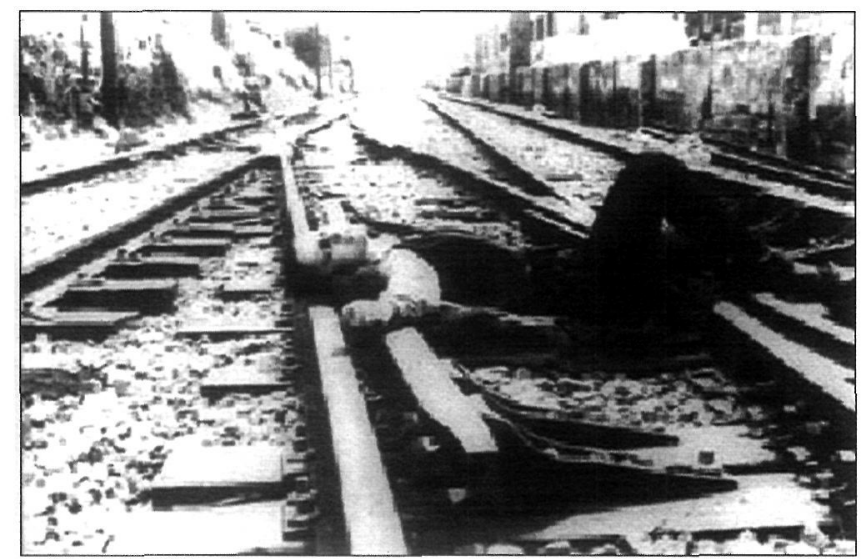

El objetivo del presente trabajo es analizar las características que definen la profesión de la enfermería en un momento histórico dado en el que aún continua vigente la dialéctica entre tecnología-humanismo y las dificultades propias de una profesión que parece estar sumida en una permanente crisis de identidad. Para salir de este impass se hace preciso desarrollar un nuevo paradigma para lo cual es, a su vez necesario, implicar a tres vertientes básicas: La metodología científica, el marco conceptual y el lenguaje profesional.

\section{AN APPROACH TO NURSING IN THE PRESENT CONTEXT}

\section{SUMMARY}

The aim of the present article is to analyse the characteristics which convey the idea of what nursing means in a concrete specific historical moment where discussions about technology versus humanism are still valid, as well as the difficulties related to a profession which seems to be sunk in a permanent identity crisis. To overcome the present impass, it is compulsory to develop a new paradigm taking into consideration three different basic points: The scientific methodology, a conceptual pattern and a professional language. 


\section{INTRODUCCIÓN}

El análisis que planteamos, sin ser exhaustivo, pretende recoger una serie de factores que de manera general caracterizan el contexto social, con grandes repercusiones en el contexto sanitario y de una manera específica y directa en Enfermería si como tal se acepta: "Un servicio de ayuda específico demandado por la sociedad". Los enfermeros como el resto de profesionales sanitarios desempeñamos un rol que va evolucionando en consonancia con las demandas y necesidades sociales, para ello estamos desarrollando nuestro propio cuerpo de conocimientos, en proceso de consolidación y de construcción de nuestra disciplina, cuyo objetivo es dar soluciones específicas y eficaces a los ciudadanos como contribución diferenciada de otras profesiones y con mejores resultados que los que puedan aportar otros.

\section{CONTEXTO SOCIAL}

Desde hace unos años estamos inmersos en un proceso de crisis social generalizada a todo el mundo, creando una situación de inestabilidad, en la que destacamos varios factores que afectan de forma global:

* Desideologización, vacío ideológico que produce una pérdida de ideas y valores que guían las conductas y el posicionamiento de las personas ante la vida.

* Redefinición del sistema socioeconómico mundial tendente a la homogeneización en torno a un marco neoliberal.

* Rearme ideológico integrista, reivindicando nuevas fronteras económicas, culturales y étnicas.* Desequilibrio entre pobreza y riqueza, incrementándose las diferencias entre países ricos y pobres, provocando fenómenos migratorios debido a guerras, miedo, violencia, hambre y deslumbrados por la publicidad de la riqueza del primer mundo. E n nuestro entorno, Occidente, habría que añadir otros más específicos:

* Cambios sociodemográficos, destacando:
- tendencia de la población a agruparse en grandes concentraciones urbanas.

- caída del índice de fecundidad; envejecimiento de la población,; incremento de la esperanza de vida.

* Crecimiento del nivel económico y aumento de la calidad de vida conjuntamente con el desarrollo de bolsas marginales.

* Asentamiento de una cultura individualista, competitiva, el culto a la imagen, la belleza, la juventud y paralelamente la devaluación y marginación de los ancianos.

* Gran cantidad de estímulos, de posibilidades, que, con frecuencia incorporamos como necesidades insatisfechas.

* Rapidez en la evolución de lo cotidiano (valores, costumbres relaciones..).

* Incorporación de la mujer como sujeto con pleno derecho en la sociedad.

* Cambios en la estructura y dinámica familiar.

*Nuevas tecnologías, "autopistas de la información", intensificando las relaciones internacionales y universalizando-homogeneizando la cultura y la formación.

* Creciente preocupación por temas ecológicos y por organizaciones no gubernamentales.

\section{CONTEXTO DEL SISTEMA SANITARIO}

En principio, se puede plantear que la finalidad de un servicio sanitario es satisfacer las necesidades de salud y las expectativas de la población, sin embargo, observamos que tienen una serie de condicionantes generales que los caracterizan y que al mismo tiempo, mediatizan su estructura y su organización, las cuales no han sido mantenidas en todas las épocas ya que el Sistema de Salud es un sistema abierto y como tal interactúa con su entorno, donde la realidad social se mantiene en continuo cambio, haciendo muy difícil el mantenimiento de normas rígidas y fijas. 
Las principales características que definen los servicios de salud en España las resumimos en:

- Un sistema que con frecuencia parece que funciona más en relación al mantenimiento de sus propias estructuras y de los intereses de los profesionales que en función de las necesidades de salud que plantean los usuarios.

- Orientación curativa y escasamente cuidadora; el objetivo fundamental es la curación por lo que en esencia su estructura y funcionamiento obedecen a este objetivo.

- Preponderancia de la tecnología sobre los aspectos relacionales, la oferta del sistema está basada en un alto grado de desarrollo tecnológico.

- Hospitalocentrismo, una arraigada cultura hospitalaria entre profesionales y población, por la que se entiende el hospital como el dispositivo de elección preferente, por ser el más cualificado.

- El elemento principal del sistema es el médico ya que es agente y proveedor y con su ejercicio profesional determina el gasto, las pruebas diagnosticas y los recursos terapéuticos que son necesarios.

- La distancia que imponen los profesionales con los usuarios, basada en el conocimiento, supedita que las decisiones las tomen los profesionales.

- Divergencia de intereses entre profesionales e instituciones con poca participación en la gestión y planificación.

- Mayor exigencia de los usuarios en cuanto a prestaciones, calidad, información y derechos y paralelamente disminución del número de cuidadores informales, que tradicionalmente han soportado una enorme carga asistencial.

asa participación del ciudadano en la gestión del si ma.

- K cursos insuficientes dedicados a la marginahdad, cubriendo estas necesidades, en gran medida, asociaciones religiosas y grupos de voluntariado.

- El cambio de las políticas sanitarias introduciendo criterios empresariales a la gestión del servicio público, por lo que se abren líneas prioritarias de actuación en torno a la identificación del producto, medición y valoración del costo y competitividad y venta del mismo.

\section{CONTEXTO ACTUAL DE ENFERMERÍA}

En la situación actual, caracterizada por valores y estructuras sociales en continuo proceso de cambio a un ritmo vertiginoso, se generan requerimientos personales y sociales, nuevas necesidades, que en muchas ocasiones no pueden ser autosatisfechas y por lo tanto necesitan cuidados, cuidados profesionales.

Al hablar de cuidados profesionales nos surgen algunas preguntas, que ya parecen clásicas, ¿la aportación, el producto enfermero es socialmente necesario, diferenciado y mejor que el de otros profesionales?, ¿utilizamos metodología científica?. Ambas cuestiones enmarcan el contexto en el que nos movemos y básicamente giran en torno a dos aspectos interdependientes: Enfermería como profesión y Enfermería disciplina científica.. Prestar cuidados, al igual que educar, es una actividad que se detecta desde los orígenes de la Historia ya que probablemente pertenezcan, ambas, a la más genuina condición del hombre como individuo que piensa y siente. Durante mucho tiempo se ha entendido la actividad enfermera (conocimientos, técnicas y práctica) como auxiliar, subsidiaria, secundaria de la actividad médica de curar. Más recientemente se tiene conciencia de que la enfermería desarrolla funciones y actividades que le son propias y exclusivas, que son complementarias pero específicas y que estando muy interrelacionada con otras disciplinas tiene identidad propia.

Sin embargo, Enfermería, a pesar de tan larga tradición histórica, con gran experiencia práctica y muchos conocimientos acumulados, no goza de un espacio epistemológico y disciplinar indiscutible, por muy diversas razones pero, fundamentalmente, por la estrecha vecindad con otras disciplinas que gozan de un status superior y por las muy variables situaciones en las que históricamente se ha tenido que desenvolver su práctica que ha estado determinada por los contextos sociales del momento. 
Es por ello que dos cuestiones, profesión y disciplina, especialmente esta última, son las que nos ocupan en la actualidad y vamos a analizar a continuación.

\section{Aspecto profesional}

Más que dar una definición profesional preferimos conceptualizarla a través de las características que la conforman:

*Colectivo que para el ejercicio de su actividad requiere una titulación específica.

*Poseer un cuerpo de conocimientos propio.

*Valores y objetivos comunes en relación al servicio que prestan.

*Cumplimiento de una función social necesaria.

*Autonomía en su campo de actuación.

*Diferenciada de otras profesiones.

*Aporta soluciones y resultados mejores que los que puedan aportar otros.

*Regulación legal de su actividad.

*Atenerse a un código deontológico.

A grandes rasgos podemos afirmar que se ha alcanzado el reconocimiento profesional: es necesaria una formación académica reglada y la obtención de un título para ejercer; está regulada legalmente, protegida contra el intrusismo y en líneas generales, entre nosotros, existe la convicción de pertenecer a un grupo. Aunque si estamos de acuerdo con la afirmación de Rosa María Alberdi: " la característica fundamental de una profesión es que sus miembros se identifiquen básicamente con una ideología común en relación al servicio que prestan", tendríamos que reconocer que en este terreno aún mantenemos diferencias entre nosotros.

En España, tres cuestiones han repercutido profundamente en el colectivo:

- Desde el año 1.977 los estudios de enfermería se imparten en la Universidad, pertenecen al ámbito de la educación superior y preparan como indica la Ley de Reforma Universitaria "para el ejercicio de actividades profesionales que exijan la aplicación de conocimientos y métodos científicos". (L.R.U. art. 1.lb.)

- Reglamentaciones legales que posibilitan el acceso a puestos de mayor responsabilidad y relevancia social, como el establecimiento de las direcciones de enfermería.

- La Ley General de Sanidad, de 1.986, donde se recoge el papel a desarrollar por los profesionales sanitarios ante la sociedad.

Los indicadores objetivos de crecimiento y desarrollo profesional son numerosos, a la integración de la enfermería en la Universidad y al acceso a los puestos de responsabilidad en gestión y administración tenemos que añadir el incremento de las actividades de formación continuada, el aumento del número de proyectos de investigación enfermeros, o la participación en proyectos multidisciplinarios, así como, la proliferación de reuniones científicas y la calidad de publicaciones propias.

Enfermería, como toda profesión, está sometida a unas normas éticas a través de un Código Deontológico; el C.I.E., en 1.953, aprobó el primer Código Ético que se revisa periódicamente y supone una declaración de principios que orienta la conducta ética de los profesionales en relación a los usuarios, a la sociedad, al ejercicio profesional y a los colegas. En relación con esto queremos recoger lo que L. Cibanal plantea: "Ética y calidad de cuidados vienen a ser lo mismo"..Sin embargo, a todos estos aspectos positivos, a los que hemos hecho referencia, hay que añadir otros aspectos negativos de la situación actual de desarrollo y consolidación profesional:

- El creciente número de enfermeros que abandonan su actividad y la significativa incidencia del "Síndrome de Burn-out".

- Limitación de la carrera profesional, con pocas posibilidades de promoción y desarrollo una vez conseguida la titulación para poder ejercer su labor profesional.. Imposibilidad de acceder a una titulación universitaria de segundo ciclo en la propia área de conocimiento. 
- El no desarrollo de las distintas especialidades salvo Matronas.

- Los problemas de la identidad profesional.

- El escaso reconocimiento social de la profesión que según refiere Adela Harto: "Sólo el que siente satisfacción en la realización de su trabajo consigue para su profesión el reconocimiento propio y de los demás".

\section{Carácter científico}

Kuhn plantea: "El progreso de la ciencia se caracteriza por ser una actividad desorganizada y diversa y se estructura y dirige finalmente cuando una comunidad científica se adhiere a un sólo paradigma. Es decir, después de una crisis de identidad se constituye una revolución, hasta que surge un nuevo paradigma lleno de promesas, que guía entonces la actividad científica normal".

Tres son las líneas en las que actualmente nos movemos en relación a la construcción de la disciplina: la metodología científica, el marco conceptual y el lenguaje profesional.

\section{Metodología científica}

Bunge recoge al respecto: "La peculiaridad de la ciencia tiene que consistir en el modo como opera para alcanzar algún objetivo determinado, o sea, en el método científico y en la finalidad para la cual se aplica dicho método".Constituye un criterio básico para el reconocimiento de una actividad o disciplina como ciencia. Supone el medio instrumental por el que la ciencia debe llegar a lograr sus objetivos como tal: describir, explicar, predecir y controlar. Estos objetivos ponen de relieve aspectos consustanciales al concepto de ciencia, como son: la dependencia de lo empírico, lo observable, la realidad; la necesidad de generar teorías explicativas e integradoras de los datos y los fenómenos en argumentos e interpretaciones coherentes con la observación; y por último, la energía transformadora de la ciencia se pone de manifiesto mediante la predicción y el control de los fenómenos a partir del conocimiento acumulado. En la década de los 50 surgen los primeros intentos de elaborar un método para resolver los problemas que planteaba el cuidado de los pacientes, (P.A.E.), la utilización del proceso, con la estructura actual, fue generalizándose en 1.973 en EE.UU cuando la Asociación Norteamericana de Enfermeras (A.N.A.) publicó las normas para la Práctica de Enfermería, con lo que se inició una revisión de su modo de trabajo en base al P.A.E.

En Europa recibió un gran impulso cuando la Oficina Regional de la O.M.S. decidió hacer del Proceso de Atención de Enfermería parte integrante de su programa europeo. Actualmente todos los teóricos están de acuerdo y así lo expresan, que la enfermería fundamenta sus actividades aplicando una metodología de trabajo uniforme, sea cual sea el modelo de referencia, el denominado P.A.E. que permite adquirir un hábito de trabajo sistemático que favorece la calidad de los cuidados, mediante un proceso de acción estructurado y flexible que posibilite tratar las cambiantes y, a veces, difíciles situaciones que se generan en torno al individuo y su proceso de salud-enfermedad. Al mismo tiempo, nos sirve para investigar, ya que, a través de él podemos conocer el impacto de las acciones de enfermería en la salud de los individuos y la calidad de los cuidados que se prestan.

\section{Marco conceptual}

Constituyen una representación estructurada de conceptos universales de interés para una disciplina, contienen el sistema filosófico y teórico predominante, proporcionan una dirección para conseguir el objetivo, es decir, el ideal que se persigue explícito en el modelo, indican las áreas de estudio que son de interés, mostrando las relaciones entre los diversos componentes y se construyen sobre la base de señalar esencialmente puntos de desarrollo que ayuden a ordenar la práctica de la disciplina configurando la esencia de la profesión. King y Browel proponen: "Sin una estructura conceptual con la que ordenar, interpretar y dar significado a los hechos, nos es imposible distinguir lo esencial de lo trivial, lo singular de lo corriente, la causa del efecto". 
La concepción de enfermería se ha ido modificando al mismo tiempo que han evolucionado los conceptos que conforman su marco de referencia. Estos cambios se han visto reflejados por los diferentes modelos de enfermería, que en función de cómo expresan e interrelacionan los conceptos de persona, salud, entorno y cuidados, ofrecen distintas formas de orientar la práctica profesional.

Los modelos, salvo el de F. Nightingale, se desarrollan a partir de la década de los 50 por lo que la construcción teórica de enfermería es muy reciente. En nuestro país el conocimiento de estos modelos, para la inmensa mayoría del colectivo profesional, no se produce hasta la década de los 80 por medio del curso de nivelación.

\section{Lenguaje profesional}

Al igual que cada individuo con sus palabras de alguna manera transmite lo que es, desde el discurso profesional transmitimos nuestra posición como enfermeras. El lenguaje profesional debe llevar implícito aquellas características que dan a la profesión enfermera una entidad específica y única, diferenciándola de cualquier otra.

La tipificación del lenguaje, incluso la jerga profesional, son elementos que dan consistencia. Una de las vías, más importante, de unificación del discurso es la utilización del Diagnóstico Enfermero, sin olvidar que es un camino recorrido por otras disciplinas en su proceso de consolidación y que otras profesiones con mayor desarrollo disciplinar todavía están en proceso de unificación de diagnósticos, como el caso de la Psiquiatría y la Psicología.

Diagnosticar es tipificar situaciones particulares que permiten la comunicación entre profesionales de forma concisa, concreta, clara y fiable por medio de significantes precisos que permiten estructurar y recuperar la información orientando las acciones pertinentes, la intervención.

El sistema de clasificación diagnóstica debe tener en cuenta:

- El mantenimiento de un criterio común y constante en base a un modelo, es decir, consistencia interna.
- Debe ser abierto y dinámico entendiendo su sentido de provisionalidad.

De los aspectos analizados en relación a la construcción de la disciplina, creemos que el de los diagnósticos es el de menor grado de desarrollo en nuestro país y el que representa mayores niveles de confusión, incluso de rechazo, no obstante, a pesar de todas las dificultades reales que presenta consideramos que es un camino imprescindible que debemos recorrer para llegar.

\section{PROPUESTAS PARA LA ACCIÓN}

Tras lo que hemos planteado entendemos que forma parte de nuestra responsabilidad sugerir algunas ideas para afrontar la situación actual y el futuro inmediato.

Aunque la mayoría de ellas están en marcha entendemos que es el momento de asumir mayores cotas de iniciativa, desarrollando las diferentes áreas:

* Trabajar por la construcción de la disciplina, a través de las tres líneas apuntadas anteriormente: modelo conceptual, metodología científica y lenguaje profesional.

*Combatir el divorcio entre " lo teórico y lo práctico ". En nuestra opinión, creemos que es mayor la obligación y la posibilidad que tenemos los docentes de facilitar puntos de encuentro, debiendo adecuar la formación de los alumnos a la realidad asistencial para lo cual se debe conocer e investigar conjuntamente con los colegas de la asistencia, pues creemos que este es uno de los métodos más eficaces para garantizar el desarrollo profesional.

*Reconocimiento profesional, para ello debemos:

- Demostrar que cumplimos una función social necesaria.

- Demostrar que dicha función es diferente a las de otras profesiones.

- Que las soluciones que aportamos son mejores.

- Que los resultados son eficientes. 
* Participar en foros ajenos a la profesión.

* Colaborar en el afianzamiento de asociaciones profesionales.

* Seguir exigiendo el desarrollo de las especialidades del decreto de 1.987 y la implantación del segundo ciclo.

* Incrementar el desarrollo de actividades científicas en la línea de resolver problemas, razonar ante nuevas situaciones y ser capaces de adaptarse y anticiparse a los cambios, dejando constancia escrita de ello, rompiendo de esta forma un aspecto de nuestra historia que ha supuesto un freno al desarrollo profesional: " la cultura profesional de la transmisión oral del saber ", porque como bien dice la profesora Collière: "Una profesión cuya historia no está escrita, es como aquel que no ha sido inscrito en el registro civil: sin archivos no se puede integrar a la sociedad a la que pertenece y no puede ser reconocido".

*Asumir la responsabilidad de la intervención de enfermería en aquellos aspectos que son invariables, suscribiendo las palabras de Rosa María Alberdi cuando dice: "Las enfermeras del siglo XXI, como ayer, como hoy y como siempre ocurrirá mientras los seres humanos seamos tan frágiles, tan insoportablemente leves, como nos define Kundera; deberán seguir siendo: la VOLUNTAD de los sin deseo; la FUERZA de los que la perdieron; el CONOCIMIENTO de los que no pueden tenerlo o de los que tienen otro porque son de otras culturas, marginales..."

\section{BIBLIOGRAFÍA}

Alberdi Castell, R.M. (1992) Las enfermeras en el III milenio. XIII Sesiones de Trabajo de la A.E.E.D. Sevilla .

Alberdi Castell, R.M. (1992) La identidad profesional de la enfermera. Rol de Enfermería, 170.

Berzosa, C. et al (1994) La economía mundial en los 90. Tendencias y desafíos. Icaria-Fuhen. Barcelona.

Bunge, M. (1989) La investigación científica. Su estrategia y su filosofía. Ariel, Madrid.

Cibanal, L. (1990) Proyecto Docente. Departamento de Enfermería Universidad de Alicante, Alicante,

Harto Castaño, A. (1992) Evolución de los cuidados enfermeros asistenciales. Momento actual. Propuesta de futuro. XIII Sesiones de trabajo de la A.E.E.D. Sevilla

Kuhn, T. (1990) La estructura de las revoluciones científicas. Fondo de Cultura Económica, México.

LEY GENERAL DE SANIDAD (1986) 14/1.986 de 25 de Abril.

LEY DE REFORMA UNIVERSITARIA (1983). 1 de Septiembre de 1.983.

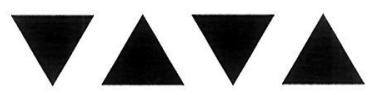

